\title{
Comparação de diferentes métodos de manejo reprodutivo em receptoras de embrião bovino sobre a taxa de concepção*
}

\section{Comparison of different reproductive management methods in bovine embryo recipients on the conception rate}

\author{
Matheus Elias de Oliveira Morais, ${ }^{* *}$ Raquel Rodrigues Costa Mello, ${ }^{* * *}$ \\ Joaquim Esquerdo Ferreira, ${ }^{* * *}$ Marco Roberto Bourg de Mello****
}

\begin{abstract}
Resumo
O objetivo deste experimento foi comparar a taxa de concepção de receptoras de embrião bovino utilizando dois diferentes métodos de manejo reprodutivo nesses animais, como a utilização do estro natural e a sincronização da ovulação para TETF. As 1802 receptoras utilizadas neste estudo foram divididas em dois grupos (controle, $n=401$ e TETF, n=1401). No grupo controle, os animais não receberam nenhum protocolo hormonal, sendo os embriões transferidos após observação do estro natural. No grupo TETF, as receptoras foram sincronizadas com protocolo hormonal iniciado em um dia aleatório do ciclo estral, com a inserção de um implante intravaginal com $1 \mathrm{~g}$ de progestágeno e administração de $2 \mathrm{mg}$ de benzoato de estradiol, sendo esse considerado o dia 0 (D0). No D8, o implante intravaginal foi removido, sendo administrados $0,15 \mathrm{mg}$ de PGF2 $\alpha, 0,5 \mathrm{mg}$ de cipionato de estradiol e 300 UI de eCG. A transferência de embriões foi realizada sete dias após a detecção do estro no grupo controle e no D17 no grupo TETF. O diagnóstico de gestação foi realizado com o auxílio de ultrassom 30 dias após a transferência. Os dados foram avaliados pelo teste do Qui-quadrado, com nível de significância de $5 \%$. Não houve diferença $(P=0,3295, P<0,05)$ entre os grupos (grupo controle: $32,7 \%$; grupo TETF: $30,1 \%$ ). Desta forma, conclui-se que a utilização dos dois diferentes métodos de manejo reprodutivo mostrou a mesma eficiência sobre a taxa de concepção em receptoras de embrião bovino.
\end{abstract}

Palavras-chave: gestação, sincronização, transferência de embrião.

\begin{abstract}
The aim of this work was to compare the conception rate of bovine embryo recipients using two different reproductive management methods in these animals, as the use of natural estrus and ovulation synchronization for FTET. The 1802 recipients were divided into two groups (control, $n=401$ and FTET, $n=1401$ ). In control group the animals received no hormonal protocol, in which natural estrus was observed. In FTET group, the recipients were synchronized by hormonal protocol started on a random day of the estrous cycle, with the insertion of an intravaginal implant with $1 \mathrm{~g}$ of progestogen and administration of 2 mg of estradiol benzoate, and this was considered day 0 (D0). On D8, the intravaginal implant was removed, and $0.15 \mathrm{mg}$ of PGF $2 \alpha, 0.5 \mathrm{mg}$ of estradiol cipionate and $300 \mathrm{IU}$ of eCG was administered. The embryo transfer was performed 7 days after estrus detection in the control group and on D17 in FTET group. Pregnancy diagnosis was performed with the aid of ultrasound 30 days after transfer. Data were analyzed by Chi-square test, with a significance level of $5 \%$. There was no significant difference $(P=0.3295, P<0.05)$ between groups $($ control group: $32.7 \%$; FTET group: $30.1 \%$ ). Thus, we can conclude that the use of two different reproductive management methods showed the same efficiency on the conception rate of bovine embryo recipients.
\end{abstract}

Keywords: embryo transfer, pregnancy, synchronization.

\section{Introdução}

A pecuária bovina brasileira tem se mostrado altamente sofisticada, com avanços tecnológicos nas áreas de reprodução e utilização de biotécnicas, como a inseminação artificial em tempo fixo (IATF), a transferência de embriões (TE) e a produção in vitro de embriões (PIV), gerando benefícios econômicos aos criadores (Buratini Jr., 2006).
A produção in vitro de embriões (PIV) é uma técnica que vem se estabelecendo em todo o mundo, principalmente no Brasil, sendo cada vez mais acessível aos criadores de bovinos (Arruda et al., 2012). No entanto, observa- se uma grande variabilidade no sucesso das transferências dos embriões bovinos produzidos in vitro, e a maioria dos problemas são relacionados com as receptoras (Andrade et al., 2012). Fatores como a idade das receptoras, grau de assincronia embrião-receptora, reutilização

*Recebido em 8 de março de 2013 e aceito em 7 de julho de 2013.

**Aluno de Graduação em Zootecnia, Departamento de Reprodução e Avaliação Animal, Instituto de Zootecnia, Universidade Federal Rural do Rio de Janeiro (UFRRJ), Km 7, BR 465, 23890-000, Seropédica, Rio de Janeiro, Brasil. Autor para correspondência.E-mail: matheusmorais@ufrrj.br

***Aluno de Pós-Graduação em Zootecnia, Departamento de Reprodução e Avaliação Animal, Instituto de Zootecnia, Universidade Federal Rural do Rio de Janeiro (UFRRJ), Km 7, BR 465, 23890-000, Seropédica, Rio de Janeiro, Brasil. E-mail: raquelmello@ufrrj.br; j.esquerdo@yahoo.com.br

****Professor Adjunto, Departamento de Reprodução e Avaliação Animal, Instituto de Zootecnia, Universidade Federal Rural do Rio de Janeiro (UFRRJ), Km 7, BR 465, 23890-000, Seropédica, Rio de Janeiro, Brasil. E-mail: mmello@ufrrj.br 
das receptoras, localização e número de corpos lúteos na receptora, condição nutricional das receptoras, controle do ciclo estral e manejo das receptoras são apontados como os de fundamental importância em programas de PIV, sendo responsáveis pelo sucesso ou falha na fertilidade dos rebanhos (Callesen et al., 1996; Hasler et al., 1987; Sreenan e Diskin, 1987; Evangelista e Sousa, 1999; Peixoto et al., 2004; Jones e Lamb, 2008).

O manejo das receptoras para a transferência dos embriões produzidos in vitro pode ser realizado por diferentes métodos, tais como por meio da observação da manifestação natural do estro, da utilização de tratamentos hormonais com intuito de sincronização do estro, e da utilização de protocolos hormonais com o intuito de sincronização da ovulação para TETF. Todos esses métodos são eficazes para aumentar o número de bezerros produzidos por embrião transferido (Jones e Lamb, 2008).

O método de observação da manifestação natural do estro apresenta algumas desvantagens como o fato de exigir um maior número de animais, depender de uma eficiente detecção do estro e não se ter controle da data da inovulação. Em contrapartida, no método de utilização de protocolos hormonais com o intuito de sincronização da ovulação para TETF, o número de animais pode ser reduzido, não há a necessidade de observação do estro, e a data da transferência poder ser programada. No entanto, há o custo adicional com hormônios (Bó et al., 2006).

Portanto, o objetivo do presente estudo foi comparar a taxa de concepção de receptoras de embrião bovino produzidos in vitro utilizando-se dois diferentes métodos de manejo reprodutivo nesses animais.

\section{Material e métodos}

O estudo foi conduzido entre dezembro de 2011 e fevereiro de 2012 na Empresa Minerembryo Reprodução e Produção Animal LTDA (Central da Receptora), que atua nas áreas de transferência de embriões (TE) e produção in vitro de embriões (PIV) em bovinos. Foram utilizadas como receptoras novilhas bovinas cruzadas de todas as raças (mestiças), procedentes da Fazenda Chapadão, em Pimenta, localizada no estado de Minas Gerais. A seleção das novilhas receptoras para as transferências dos embriões produzidos in vitro foi feita observando-se características de docilidade, facilidade de parto e avaliação do trato reprodutivo, eliminando os animais que apresentavam cervix fibrosa ou tortuosa.

As receptoras apresentavam exames negativos para brucelose, tuberculose e de leucose enzoótica. Estas foram vacinadas por duas vezes contra rinotraqueíte infecciosa bovina (IBR), diarreia viral bovina (BVD), leptospirose, carbúnculo, raiva e febre aftosa. Com relação ao manejo nutricional, elas foram mantidas a pasto de capim Brachiaria, onde receberam também suplementação mineral no cocho e água à vontade.

Para a produção dos embriões, foi utilizada a técnica de Ovum Pick Up (OPU), em que foram coletados complexos cumulusoócitos (CCOs) in vivo de uma fêmea doadora, procedentes de clientes da Empresa. Essa Empresa não tem laboratório específico: estabelece parceria com outros laboratórios, onde os técnicos vão até a fazenda dos clientes, coletam os CCOs e produzem os embriões in vitro, enviando-os para a Empresa. Esta, por sua vez, transfere e entrega as receptoras gestantes para os clientes. Os embriões foram produzidos a partir de cruzamentos entre Jersey e Holandês (vaca Jersey $x$ touro Holandês).

Para a realização da OPU, foi utilizado um aparelho de ultrassom (MINDRAY DP 2200Vet) e uma probe convexa de $5 \mathrm{MHz}$ adaptada a uma guia para a aspiração folicular transvaginal. Após a aspiração, o conteúdo aspirado foi lavado e filtrado em filtro próprio e vertido em placas de Petri para realização da procura, manipulação e avaliação morfológica dos CCOs. A avaliação morfológica foi realizada segundo Leibfried e First (1979), levando-se em consideração características das células da granulosa e do citoplasma do oócito. Foram considerados viáveis apenas CCOs classificados como graus I, II e III.

Após esta etapa, os CCOs passaram pelas etapas de maturação, fecundação e cultivo in vitro e, no oitavo dia, os embriões classificados como viáveis foram envasados em palhetas de $0,25 \mathrm{~mL}$, sendo transferidos a fresco para receptoras que não receberam nenhum tipo de protocolo hormonal (observação natural do estro) ou para receptoras sincronizadas por meio de protocolo hormonal (protocolo de sincronização da ovulação para TETF).

Os embriões foram transferidos para 1802 novilhas bovinas receptoras. Essas receptoras foram divididas em dois grupos, conforme o manejo utilizado para a transferência dos embriões (grupo Controle; n=401 e grupo TETF - Transferência de Embrião em Tempo Fixo; n=1401). No grupo controle (observação da manifestação natural do estro), os animais não receberam nenhum tipo de protocolo hormonal, sendo a transferência dos embriões realizada sete dias após a observação de estro natural (D7). No grupo TETF (protocolo de sincronização da ovulação para TETF), as receptoras foram sincronizadas por protocolo hormonal iniciado em um dia aleatório do ciclo estral, com a colocação de um implante intravaginal com $1 \mathrm{~g}$ de progestágeno e administração de $2 \mathrm{mg}$ de benzoato de estradiol, sendo este considerado o dia 0 (D0). No D8, foi removido o implante intravaginal e administrado $0,15 \mathrm{mg}$ de análogo de PGF2 $\alpha$ (D-cloprostenol), 0,5 mg de cipionato de estradiol e $300 \mathrm{UI}$ de eCG Gonadotrofina Coriônica Equina), sendo a transferência dos embriões realizada no D17 (Nasser et al., 2004).

A transferência dos embriões foi realizada com auxílio de inovulador, sendo colocado na parte mais cranial do corno uterino ipsilateral ao ovário que continha o corpo lúteo. $\mathrm{O}$ diagnóstico de gestação foi realizado com auxílio de um aparelho de ultrassom (MINDRAY DP 2200Vet) 30 dias após a transferência embrionária.

A análise estatística relacionada com a comparação da taxa de concepção entre os grupos foi realizada com o auxílio do teste do Qui-quadrado, com nível de significância de 5\% $(P<0.05)$.

\section{Resultados e discussão}

As taxas de concepção para os grupos controle e TETF foram $32,7 \%$ e $30,1 \%$, respectivamente. Pela análise dos dados, observou-se que não houve diferença $(P=0,3295 ; P>0,05)$ entre os grupos avaliados. Em um programa de transferência de embriões (TE) ou de produção in vitro de embriões (PIV), os embriões podem ser transferidos para as receptoras durante um ciclo estral normal, ou, de maneira mais prática, durante um ciclo estral controlado por meio de hormônios para realização da TETF (Bó et al., 2002). A questão do estro induzido por hormônios ser menos eficiente para a subsequente transferência de embriões, quando comparado ao estro natural, ainda 
permanece controverso (Callesen et al., 1996). Na literatura, as taxas de concepção de receptoras de embrião bovino que tiveram o estro induzido comparado ao estro natural têm sido relatadas como altas (Hasler et al., 1987), baixas (Weaver et al., 1986) ou equivalentes (Wright, 1981; Callensen et al., 1994).

No presente estudo, não houve diferença nas taxas de concepção entre os grupos de manejo (estro natural e TETF), sendo que, dessa maneira, os diferentes métodos de preparo das receptoras poderão ser utilizados conforme suas vantagens (benefícios) e desvantagens (limitações). A vantagem da utilização do estro natural é a redução dos custos com hormônios. No entanto, são necessárias pessoas treinadas para a detecção do estro, maior número de receptoras, e maior flexibilidade do profissional que realizará a inovulação, uma vez que a data da transferência não é controlada (depende do dia do estro).

Os métodos disponíveis para o tratamento de receptoras de embriões bovinos incluem a observação natural do estro, a indução do estro com o uso da prostaglandina F2 $\alpha$ (PGF2 $\alpha$ ) ou seus análogos sintéticos e o emprego de hormônios para a sincronização da ovulação para TETF. A utilização de análogos da PGF2a para a indução do estro é um dos métodos menos dispendiosos para obtenção de receptoras aptas à transferência de embriões (Mariani, 2009).

No entanto, existem algumas condições imprescindíveis para o seu uso, tais como a detecção do corpo lúteo por palpação retal ou ultrassonografia e a observação do comportamento estral nos dias subsequentes ao tratamento (Barreiros et al., 2006; Mariani, 2009). Além disso, em um grupo de receptoras tratadas com PGF2 $\alpha$ associado à detecção do estro, somente de 40 a $50 \%$ dos animais tratados são usados para a transferência de embriões. Considerando as taxas de concepção de aproximadamente $50 \%$ em todos os animais tratados, somente de 20 a $25 \%$ das prenhezes são obtidas ao final dos programas de transferência de embriões (Bó et al., 2002; Marques et al., 2004; Baruselli et al., 2005).

Os estudos encontrados na literatura relacionados com as taxas de concepção entre receptoras manejadas por meio do estro natural ou induzido apresentam resultados conflitantes (Callesen et al., 1996). Cairoli et al. (2006) demonstraram não haver diferenças nas taxas de concepção comparando o estro espontâneo ou o estro induzido por PGF2a. Bényei et al. (2006) também não observaram diferenças nas taxas de concepção de receptoras tratadas por meio do estro natural ou do estro sincronizado com PGF2 $\alpha$ (40,9 e 41,8\%, respectivamente, $\mathrm{P}=0,598)$. Por outro lado, Hasler et al. (1987) observaram uma maior taxa de concepção em receptoras tratadas com PGF2a em relação àquelas manejadas por observação natural do estro (75,0 versus $68,0 \%$, respectivamente, $P<0,25)$.

Atualmente, a alternativa mais útil para se aumentar de modo significativo o número de animais envolvidos em programas de TE ou PIV é a aplicação de protocolos hormonais que permitem a transferência de embriões para as receptoras sem a necessidade de detecção de estro, geralmente chamados de protocolos de transferência de embriões em tempo fixo (TETF, Bó et al., 2002; Bó et al., 2006; Looney et al., 2006; Jones e Lamb, 2008), pois são capazes de proporcionar um incremento nas taxas de aproveitamento das receptoras, e com isso, elevadas taxas de concepção em relação ao número de animais tratados (Marques et al., 2004; Baruselli et al., 2005). De acordo com Barreiros et al. (2006), além de facilitar consideravelmente o manejo, as taxas de aproveitamento estão em torno de $70 \%$ dos animais tratados para protocolos de TETF.

Nesse sentido, diversos trabalhos encontrados na literatura apresentam comparações entre a manifestação natural do estro e o uso de protocolos de sincronização da ovulação para TETF. Bó et al. (2004) e Baruselli et al. (2011) observaram que receptoras tratadas com protocolo de sincronização da ovulação para TETF apresentaram taxas de concepção comparáveis com as obtidas em receptoras transferidas sete dias após a detecção do estro. Assim, têm sido relatadas taxas de concepção semelhantes ou maiores em receptoras tratadas com protocolo para TETF em comparação àquelas observadas após a detecção de estros espontâneos, mas as taxas de concepção têm melhorado porque esses protocolos têm aumentado o número de receptoras que receberam um embrião entre as tratadas (Bó et al., 2002).

Colombo et al. (2010) não observaram diferenças nas taxas de concepção ao avaliarem 1590 novilhas mestiças receptoras tratadas com implante de progestágeno e benzoato de estradiol ou por meio da observação natural do estro $(35,15$ versus $34,47 \%, P>0,05)$. Pieroni (2009) não encontrou diferenças nas taxas de concepção ao avaliar 315 novilhas mestiças receptoras tratadas por observação natural do estro ou por meio de protocolo de sincronização da ovulação para TETF $(18,52$ versus $11,54 \%$, respectivamente, $P=0,68)$, tal como foi observado no presente trabalho.

Diversos outros trabalhos encontrados na literatura têm demonstrado comparações entre protocolos de sincronização do estro (uso somente de PGF2 $\alpha$ ) e da ovulação para TETF (uso de GnRH e PGF2 $\alpha$, ou de CIDR $^{\circledR}$, benzoato de estradiol e PGF2 $\alpha$ ), apresentando, muitas vezes, resultados inconclusivos, demonstrando diferenças ou semelhanças nas taxa de concepção obtidas (Pieroni, 2009). Tais resultados poderiam ser explicados pelas diferenças relacionadas com as receptoras, como raça, idade, grupo genético e aptidão econômica, e pelas diferentes condições de manejo experimentais, o que pode dificultar as comparações entre as diferentes metodologias.

Bó et al. (2002) não obtiveram diferenças nas taxas de concepção entre receptoras tratadas com CIDR $^{\circledR}$ para TETF ou tratadas somente com PGF2 $\alpha$ para sincronização do estro $(32,0$ versus $37,0 \%$, respectivamente, $\mathrm{P}>0,6)$. Entretanto, Baruselli et al. (2003) obtiveram melhores taxas de concepção quando utilizaram somente a PGF2 $\alpha$ em comparação com um protocolo para TETF. Looney et al. (2006) também obtiveram maiores taxas de concepção com o uso da PGF2a em comparação com um protocolo para TETF $(63,0$ versus $53,0 \%$, respectivamente, $\mathrm{P}<0,01)$.

Os resultados de Baruselli et al. (2003) e Looney et al. (2006) podem ser atribuídos ao fato de ovulações naturais, ou após a administração de PGF2 $\alpha$, ocorrerem a partir de um folículo de maior diâmetro, uma vez que esse tem que produzir estradiol suficiente para induzir o pico de $\mathrm{LH}$ e consequentemente a ovulação. Além disso, folículos de maior diâmetro resultam em maiores corpos lúteos que produzirão mais progesterona, hormônio essencial para estabelecimento da prenhez. Ao utilizar um indutor da ovulação em protocolos para TETF, normalmente essa ocorre a partir de um folículo de menor diâmetro (Pieroni, 2009). Além disso, a duração de exposição ao implante de progestágeno (como o $\mathrm{CIDR}^{\circledR}$ ) pode afetar a fertilidade das receptoras, interferindo nas taxas de concepção (Looney et 
al., 2006). De outra forma, Baruselli et al. (2011) obtiveram maiores taxas de concepção em receptoras tratadas com um protocolo para TETF em comparação com aquelas tratadas com um protocolo usando somente PGF $2 \alpha(29,3$ versus $16,2 \%$, respectivamente, $\mathrm{P}=0,001)$.

Modificações recentes incorporadas aos protocolos hormonais, como as mudanças no momento em que a PGF2 $\alpha$ é aplicada e a administração de eCG também têm melhorado as taxas de concepção, pois tem proporcionado um aumento no crescimento folicular e no diâmetro máximo do folículo dominante, com consequente aumento nas concentrações plasmáticas de progesterona no ciclo estral subsequente (Baruselli et al., 2010a). Vários trabalhos mostraram efeito positivo da progesterona no desenvolvimento embrionário e na manutenção da gestação (Binelli et al., 2001; Thatcher et al., 2001). Além disso, esses protocolos oferecem novas ideias para tornar a execução desses tratamentos mais fácil para os funcionários das fazendas através da redução do número de manejos necessários para a realização da TETF (Baruselli et al., 2010b; Bó et al., 2006).

Bó et al. (2002) relataram uma melhora nas taxas de concepção em receptoras com múltiplos corpos lúteos e aumento nas concentrações plasmáticas de progesterona induzidos por eCG, em comparação com as receptoras que não receberam eCG ( $45 \%$ versus $10 \%$, respectivamente, $P<0,05$ ). Segundo esses autores, a onda folicular foi sincronizada pela administração de progesterona e estradiol no dia 0, eCG e PGF2 $\alpha$ no dia 5 , remoção do implante de progestágeno no dia 8 , benzoato de estradiol no dia 9, e TETF no dia 17. Baruselli et al. (2001) também demonstraram que os tratamentos com eCG administrados quatro dias antes do estro aumentaram o número de ovulações, as concentrações plasmáticas de progesterona, e as taxas de concepção em receptoras Bos indicus cruzadas sincronizadas com dispositivos de liberação de progesterona, benzoato de estradiol, e progesterona, e transferidas sem a detecção do estro.

Apesar de não ter havido diferença entre o uso do estro natural ou o protocolo hormonal no presente trabalho, o uso de protocolos hormonais para TETF torna-se mais vantajoso devido ao fato de poder se concentrar os trabalhos de manejo com os animais em momentos predeterminados, dispensando a necessidade de detecção do estro, atividade que se constitui em um dos grandes problemas na maioria das propriedades brasileiras, reduzir o intervalo entre o tratamento e a prenhez, e possibilitar a transferência em dias predeterminados (Bó et al., 2006; Baruselli et al., 2011).

Dessa forma, essa metodologia de tratamento das receptoras pode ser uma alternativa para se racionalizar o uso destes animais por meio do aumento das taxas de aproveitamento das receptoras e das taxas de concepção, aumentando também a genética e o retorno econômico dos programas de transferência de embriões produzidos in vivo (TE) ou in vitro (PIV), determinando um maior número de bezerros nascidos ao ano e reduzindo os custos com as receptoras não prenhes (Marques et al., 2004; Baruselli et al., 2005).

\section{Conclusão}

Podemos concluir que os dois diferentes métodos utilizados mostraram a mesma eficiência em relação à taxa de concepção, sendo que ambas as metodologias podem ser utilizadas com sucesso para o manejo de receptoras de embrião bovino em programas de PIV.

\section{Agradecimentos}

Os autores deste artigo científico agradecem ao Instituto de Zootecnia e ao Departamento de Reprodução e Avaliação Animal da Universidade Federal Rural do Rio de Janeiro, pelo suporte científico a esta pesquisa para a realização do trabalho de Monografia do primeiro autor; e à Empresa Minerembryo Reprodução e Produção Animal Ltda., pela concessão dos dados e o suporte à infraestrutura para este estudo.

\section{Referências}

ANDRADE, G.A.; FERNANDES, M.A.; KNYCHALA, R.M.; PEREIRA JUNIOR, M.V.; OLIVEIRA, A.J.; NUNES, D.P.; BONATO, G.L.; SANTOS, R.M. Fatores que afetam a taxa de prenhez de receptoras de embriões bovinos produzidos in vitro. Revista Brasileira de Reprodução Animal, v. 36, n. 1, p. 66-69, 2012.

ARRUDA, R.P.; CELEGHINI, E.C.C.; ALONSO, M.A.; CARVALHO, H.F.; LEMES, K.M.; SILVA, D.F.; RODRIGUEZ, S.A.F.; AFFONSO, J.F. Aspects related to the technique and the utilization of sexed semen in vivo and in vitro. Animal Reproduction, v. 9, n. 3, p. 345353, 2011.

BARREIROS, T.R.R.; BLASCHI, W.; BORSATO, E.A.; LUDWIG, H.E.; SILVA, D.R.M.; SENEDA, M.M. Comparação das taxas de prenhez entre receptoras com corpos lúteos cavitários ou compactos após protocolos de sincronização com clorprostenol ou transferência de embriões em tempo fixo. Semina: Ciências Agrárias, v. 27, n. 4, p. 657-664, 2006.

BARUSELLI, P.S.; MARQUES, M.O.; MADUREIRA, E.H.; COSTA NETO, W.P.; GRANDINETTIE, R.R.; BÓ, G.A. Increased pregnancy rates in embryo recipients treated with CIDR-B devices. Theriogenology, v. 55, p. 355, 2001 (Abstract).
BARUSELLI, P.S.; MARQUES, M.O.; CARVALHO, N.A.T.; BERBER, R.C.A.; VALENTIM, R.; CARVALHO FILHO, A.F.; COSTA NETO, W.P. Dinâmica folicular e taxa de prenhez em novilhas receptoras de embrião (Bos taurus indicus $x$ Bos taurus taurus) tratadas com o protocolo "Ovsynch" para inovulação em tempo fixo. Brazilian Journal of Veterinary Research and Animal Science, v. 40 (Suplemento 2), p. 96-106, 2003.

BARUSELLI, P.S.; MARTINS, C.M.; SÁ FILHO, M.F.; NASSER, L.N.; GIMENES, L.U.; MADUREIRA, E.H.; BÓ, G.A. Novos avanços nos tratamentos de doadoras e de receptoras de embrião bovino. Acta Scientiae Veterinariae, v. 33 (Suplemento 1), p.151156, 2005.

BARUSELLI, P.S.; SALES, J.N.S.; SÁ FILHO, M.F. Atualização dos protocolos de IATF e TETF. In: IV SIMPÓSIO INTERNACIONAL DE REPRODUÇÃO ANIMAL APLICADA, 2010. Anais... Londrina, PR: 2010a, p. 174-176.

BARUSELLI, P.S.; FERREIRA, R.M.; SÁ FILHO, M.F.; NASSER, L.T.F.; RODRIGUES, C.A.; BÓ, G.A. Bovine embryo transfer recipient synchronization and management in tropical environments. Reproduction, Fertility and Development, v. 22, p. 67-74, 2010b. 
BARUSELLI, P.S.; FERREIRA, R.M., SALES, J.N.S.; GIMENES, L.U.; SÁ FILHO, M.F.; MARTINS, C.M.; RODRIGUES, C.A.; BÓ, G.A. Timed embryo transfer programs for management of donor and recipient cattle. Theriogenology, v. 76, p.1583-1593, 2011.

BÉNYEI, B.; KOMLÓSI, I.; PÉCSI, A.; POLLOTT, G.; MARCOS, C.H.; CAMPOS, A.O.; LEMES, M.P. The effect of internal and external factors on bovine embryo transfer results in a tropical environment. Animal Reproduction Science, v. 93, p. 268-279, 2006.

BINELLI, M.; THATCHER, W.W.; MATTOS, R.; BARUSELLI, P.S. Antiluteolytic strategies to improve fertility in cattle. Theriogenology, v. 56, p.1451-1463, 2001.

BÓ, G.A.; BARUSELLI, P.S.; MORENO, D.; CUTAIA, L.; CACCIA, M.; TRÍBULO, R.; TRÍBULO, H.; MAPLETOFT, R.J. The control of follicular wave development for self-appointed embryo transfer programs in cattle. Theriogenology, v. 57, p. 53-72, 2002.

BÓ, G.A.; MORENO, D.; CUTAIA, L.; BARUSELLI, P.S.; REIS, E.L. 2004. Manipulação hormonal do ciclo estral em doadoras e receptoras de embrião bovino. Acta Scientiae Veterinariae, v. 32 (Suplemento 1 ), p.1-22, 2004.

BÓ, G.A.; PICINATO, D.; PERES, L.; CUTAIA, L.; NASSER, L.F.; BARUSELLI, P.S. Protocolos de transferência de embriões em tempo fixo para receptoras de embriões bovinos. Acta Scientiae Veterinariae, v. 34 (Suplemento 1), p. 17-23, 2006.

BURATINI Jr., J. Foliculogênese em bovinos. In: II SIMPÓSIO INTERNACIONAL DE REPRODUÇÃO ANIMAL APLICADA, 2006. Anais... Londrina, PR, 2006, p. 55-62.

CAIROLI, F.; MOLLO, A.; VERONESI, M.C.; RENAVILLE, B.; FAUSTINI, M.; BATTOCCHIO, M. Comparison between cloprostenol-induced and spontaneous oestrus fertility in dairy cows. Reproduction in Domestic Animals, v. 41, p. 175-179, 2006.

CALLESEN, H.; BAK, A.; GREVE, T. Embryo recipients: Dairy cows or heifers? Proceedings of the $10^{\text {th }}$ Scientific Meeting of the Association European Transfer Embryo, September 1994, Lyon, France. Fondation Meriuex, Lyon, 1994, p. 125-135.

CALLESEN, H.; LIBORIUSSEN, T.; GREVE, T. Practical aspects of multiple ovulation-embryo transfer in cattle. Animal Reproduction Science, v. 42, p. 205-214, 1996.

COLOMBO, A.H.B.; ZANIBONI, L.; CAVALIERI, F.L.B.; RIGOLON L.P. Estimação dos fatores que interferem diretamente e indiretamente nos resultados da fecundação in vitro (FIV). In: V MOSTRAINTERNADE TRABALHOS DE INICIAÇÃO CIENTÍFICA, 2010. Anais... Maringá, PR, 2010.

EVANGELISTA, J.J.F.; SOUSA, R.V. Resposta superovulatória e produção de embriões em vacas da raça Guzerá leiteiro. Arquivo da Faculdade de Veterinária UFRGS, v. 27, n.1, 1999. (Resumo).

HASLER, J.F.; MCCAULEY, A.D.; LATHROP, W.F.; FOOTE, R.H. Effect of donor-embryo-recipient interactions on pregnancy rate in a large-scale bovine embryo transfer program. Theriogenology, $\mathrm{v}$. 27, n.1, p.139-168, 1987.
JONES, A.L.; LAMB, G.C. Nutrition, synchronization, and management of beef embryo transfer recipients. Theriogenology, v. 69, p. 107-115, 2008.

LEIBFRIED, L.; FIRST, N.L. Characterization of bovine follicular oocytes and their ability to mature in vitro. Journal of Animal Science, v. 48, p. 76-86, 1979.

LOONEY, C.R.; NELSON, J.S.; SCHNEIDER, H.J.; FORREST, D.W. Improving fertility in beef cows recipients. Theriogenology, v. 65, p. 201-209, 2006.

MARIANI, A.C.B. Influência do dia do estro e em relação à FIV e da morfologia do corpo lúteo no dia da inovulação com os índices de prenhez em receptoras de embriões bovinos produzidos in vitro. 2009, 53 p. Dissertação de Mestrado. Universidade Federal do Tocantins, 2009.

MARQUES, M.O.; REIS, E.L.; BARUSELLI, P.S. Sincronização de receptoras para inovulação em tempo fixo. In: I SIMPÓSIO INTERNACIONAL DE REPRODUÇÃO ANIMAL APLICADA, 2004. Anais... Londrina, PR, 2004, p. 199-211.

NASSER, L.F.T.; REIS, E.L.; OLIVEIRA, M.A.; BÓ, G.A.; BARUSELLI, P.S. Comparison of four synchronization protocols for fixed-time bovine embryo transfer in Bos indicus $X$ Bos taurus recipients. Theriogenology, v. 62, p.1577-1584, 2004.

PEIXOTO, M.G.C.D.; BERGMANN, J.A.G.; ALVIM, M.T.T.; PENNA, V.M. Fatores que influenciaram a prenhez de embriões zebuínos em receptoras mestiças. In: SIMPÓSIO DA SOCIEDADE BRASILEIRA DE MELHORAMENTO ANIMAL, v. 5, 2004. Anais... Pirassununga, SP: SBMA, 2004, 4 p.

PIERONI, J.S.P. Influência do local de inovulação de embriões produzidos in vivo e in vitro sobre as taxas de concepção de fêmeas bovinas e sua relação com a morfologia uterina. 2009. 121 p. Dissertação de Mestrado em Medicina Veterinária. Faculdade de Ciências Agrárias e Veterinárias. Universidade Estadual Paulista Júlio de Mesquita Filho (UNESP), 2009.

SREENAN, J.M.; DISKIN, M.G. Factors affecting pregnancy rate following embryo transfer in the cow. Theriogenology, v. 27, p. 99113, 1987.

THATCHER, W.W.; MOREIRA, F.; SANTOS, J.E.P.; MATTOS, R.C.; LOPEZ, F.L.; PANCARCI, S.M. Effects of hormonal treatments on reproductive performance and embryo production. Theriogenology, v. 55, p. 75-90, 2001.

WEAVER, L.D.; GALLAND, J.; SOSNIK, U.; COWEN, P. Factors affecting embryo transfer success in recipient heifers under field conditions. Journal of Dairy Science, v. 69, p. 271 I-2717, 1986.

WRIGHT, J.M. Non-surgical embryo transfer in cattle: Embryorecipient interactions. Theriogenology, v.15, p. 43-56, 1981. 